\title{
Missing value imputation for microarray data: a comprehensive comparison study and a web tool
}

\author{
Chia-Chun Chiu, Shih-Yao Chan, Chung-Ching Wang, Wei-Sheng Wu \\ From 24th International Conference on Genome Informatics (GIW 2013) \\ Singapore, Singapore. 16-18 December 2013
}

\begin{abstract}
Background: Microarray data are usually peppered with missing values due to various reasons. However, most of the downstream analyses for microarray data require complete datasets. Therefore, accurate algorithms for missing value estimation are needed for improving the performance of microarray data analyses. Although many algorithms have been developed, there are many debates on the selection of the optimal algorithm. The studies about the performance comparison of different algorithms are still incomprehensive, especially in the number of benchmark datasets used, the number of algorithms compared, the rounds of simulation conducted, and the performance measures used.
\end{abstract}

Results: In this paper, we performed a comprehensive comparison by using (I) thirteen datasets, (II) nine algorithms, (III) 110 independent runs of simulation, and (IV) three types of measures to evaluate the performance of each imputation algorithm fairly. First, the effects of different types of microarray datasets on the performance of each imputation algorithm were evaluated. Second, we discussed whether the datasets from different species have different impact on the performance of different algorithms. To assess the performance of each algorithm fairly, all evaluations were performed using three types of measures. Our results indicate that the performance of an imputation algorithm mainly depends on the type of a dataset but not on the species where the samples come from. In addition to the statistical measure, two other measures with biological meanings are useful to reflect the impact of missing value imputation on the downstream data analyses. Our study suggests that local-least-squares-based methods are good choices to handle missing values for most of the microarray datasets.

Conclusions: In this work, we carried out a comprehensive comparison of the algorithms for microarray missing value imputation. Based on such a comprehensive comparison, researchers could choose the optimal algorithm for their datasets easily. Moreover, new imputation algorithms could be compared with the existing algorithms using this comparison strategy as a standard protocol. In addition, to assist researchers in dealing with missing values easily, we built a web-based and easy-to-use imputation tool, MissVIA (http://cosbi.ee.ncku.edu.tw/MissVIA), which supports many imputation algorithms. Once users upload a real microarray dataset and choose the imputation algorithms, MissVIA will determine the optimal algorithm for the users' data through a series of simulations, and then the imputed results can be downloaded for the downstream data analyses.

\footnotetext{
* Correspondence: wessonwu@mail.ncku.edu.tw

Department of Electrical Engineering, National Cheng Kung University, No.1 University Road, 701 Tainan, Taiwan (R. O. C.)
}

(C) 2013 Chiu et al.; licensee BioMed Central Ltd. This is an Open Access article distributed under the terms of the Creative Commons 


\section{Background}

Gene expression microarray (DNA chip) technology is a powerful tool for modern biomedical research. It could monitor relative expression of thousands of genes under a variety of experimental conditions. Therefore, it has been used widely in numerous studies over a broad range of biological disciplines, such as cell cycle regulation, stress responses, cancer diagnosis, functional gene discovery, specific therapy, and drug dynamic identification [1-9]. Although microarray technology has been used for several years, expression data still contain missing values due to various reasons such as scratches on the slide, spotting problems, poor hybridization, inadequate resolution, fabrication errors and so on.

Basically, microarray data contain 1-10\% missing values that could affect up to $95 \%$ of genes [10]. The occurrence of missing values in microarray data disadvantageously influences downstream analyses, such as discovery of differentially expressed genes $[11,12]$, construction of gene regulatory networks $[13,14]$, supervised classification of clinical samples [15], gene cluster analysis $[10,16]$, and biomarker detection.

One straightforward solution to solve the missing value problem is to repeat the microarray experiments, but that is very costly and inefficient. Another solution is to remove genes (rows) with one or more missing values before downstream analysis, but it is easily seen that part of important information would be lost. Hence, advanced algorithms must be developed to accurately impute the missing values.

Using modern mathematical and computational techniques can effectively impute missing values. Early approaches included replacing missing values by zero, row average or row median [17]. Recently, many studies found that merging information from various biological data can significantly improve the missing values estimation. Liew et al. categorized the existing algorithms into four different classes: (1) local algorithms, (2) global algorithms, (3) hybrid algorithms, and (4) knowledge assisted algorithms $[18,19]$.

The first category includes $k$ nearest neighbors (KNN) [17], iterative $k$ nearest neighbors (IKNN) [20], sequential $k$ nearest neighbors (SKNN) [21], least squares adaptive (LSA) [22], local least squares (LLS) [23], iterative local-least-squares (ILLS) [24], sequential local-leastsquares (SLLS) [25], and etc. The second category includes Bayesian principal component analysis (BPCA) [26], singular value decomposition (SVD) [17], partial least squares (PLS) and so on. The third category includes LinCmb [11]. The fourth category integrates domain knowledge (Gene Ontology [27] and multiple external datasets [18]) or external information into the imputation process. Projection onto convex sets (POCS)
[28], GOimpute, histone acetylation information aided imputation (HAIimpute) [29], weighted nearest neighbors imputation (WeNNI) [30] and integrative missing value estimation (iMISS) [31] belong to the knowledge assisted approach algorithms. In this study, we did not use the hybrid algorithms and the knowledge assisted algorithms because their programs are not freely available or cannot be easily modified.

In the past few years, several papers have preliminary and objective analyses for the systematic evaluation of different imputation algorithms [32-35]. The weaknesses of these studies are as follows. First, few microarray datasets were used [32]. Second, few independent rounds of the imputed procedure were performed (usually 10 times). Third, single performance measure was used $[33,34]$. Here, we present a fair and comprehensive evaluation to assess the performances of different imputation algorithms on different datasets using different performance measures.

\section{Methods}

\section{Datasets}

Considering that datasets from different species and types of datasets may have different effects on the performance of imputation algorithms, we chose thirteen different datasets from two species (Saccharomyces cerevisiae and Homo sapiens), which could be categorized into three different types (time series, non-time series and mixed type), for our analyses.

For time series datasets, we selected the yeast cell cycle data (including the alpha factor arrest and elutriation datasets) from [36], and Shapira04A and Shapira04B datasets, which were two different time series datasets (both measured the effect of oxidative stress on the yeast cell cycle) from [37]. We also chose the human cell cycle data called Human HeLa from [38]. For non-time series datasets, we chose the datasets (Ogawa, BohenSH and BohenLC) from [39] and [40]. Ogawa's data was retrieved from the study of phosphophate accumulation and poly-phosphophate metabolism and the BohenSH was retrieved from follicular lymphoma lymph node and normal lymph node and spleen samples on $\mathrm{SH}$ microarrays and the BohenLC was retrieved from 24 independent follicular lymphoma lymph node samples on LC microarrays. For mixed type datasets, we chose the datasets from Lymphoma [41] (focused on two experimental subsets corresponding to Blood B cells and Thymic T cells), Baldwin [42], Yoshimoto02 [43], Brauer05 [44] and Ronen05 [45].

Before analyses, we removed all genes with missing values to create complete matrices. And then multiple entries with different missing rates $(1 \%, 5 \%, 10 \%, 15 \%$ and $20 \%$ ) were randomly introduced into these complete 
Table 1 Benchmark datasets.

\begin{tabular}{ccccc}
\hline & & \multicolumn{3}{c}{ Datasets } \\
\hline Name & Full Dim. & Used Dim. & Category & Species \\
\hline Ogawa & $6263^{*} 8$ & $3069^{*} 8$ & Non-time series & S.cerevisiae \\
Brauer05 & $6133^{*} 60$ & $706^{*} 60$ & Mixed type & S.cerevisiae \\
Ronen05 & $6987^{*} 26$ & $2998^{*} 26$ & Mixed type & S.cerevisiae \\
Yoshimoto02 & $6166^{*} 24$ & $4380^{*} 24$ & Mixed type & S.cerevisiae \\
Spahira04A & $4771^{*} 23$ & $2970^{*} 23$ & Time series & S.cerevisiae \\
Spahira04B & $4771^{*} 14$ & $3340^{*} 14$ & Time series & S.cerevisiae \\
Spellman ELU & $6178^{*} 14$ & $5766^{*} 14$ & Time series & S.cerevisiae \\
Spellman AFA & $6178^{*} 18$ & $4489^{*} 18$ & Time series & S.cerevisiae \\
BohenSH & $2364^{*} 24$ & $623^{*} 24$ & Non-time series & H.sapiens \\
BohenLC & $13121^{*} 24$ & $615^{*} 24$ & Non-time series & H.sapiens \\
Lymphoma & $4026^{*} 16$ & $2209^{*} 16$ & Mixed type & H.sapiens \\
Baldwin & $16838^{*} 39$ & $6850^{*} 39$ & Mixed type & H.sapiens \\
Human HeLa & $1134^{*} 19$ & $920^{*} 19$ & Time series & H.sapiens \\
\hline
\end{tabular}

matrices. A brief information of these datasets is presented in Table 1.

\section{Collection of missing value imputation algorithms}

In this paper, we present a comprehensive evaluation on the performance of nine imputation algorithms on a wide variety of types and sizes of microarray datasets. We assessed the performance of different algorithms on each dataset. Algorithms used can be divided into two categories: local imputation algorithms and global imputation algorithms.

Local imputation algorithms select a group of genes with the highest relevance (using Euclidian distance $[17,23]$, Pearson correlation [22,23], or covariance estimate [46]) to the target gene to impute missing values. For local imputation algorithms, we used k-NearestNeighbors (KNN), iterative k-Nearest-Neighbors (IKNN), sequential k-Nearest-Neighbors (SKNN), least squares adaptive (LSA), local least squares (LLS), iterative LLS (ILLS) and sequential LLS (SLLS). For global imputation algorithms, we used singular value decomposition
(SVDimpute) and Bayesian principal components analysis (BPCA). The KNN and SVD algorithms were run with the parameter $k=15$, the SKNN algorithm was run with the parameter $k=10$ for time series data and $k=15$ for non-time series data. The automatic parameter estimator was used for LLS, SLLS and BPCA. The LS, IKNN and ILLS methods do not contain any free parameters. A brief information of these algorithms being used is presented in Table 2.

\section{Performance indices}

We used three performance indices (normalized root mean squared error, cluster pair proportions and biomarker list concordance index) to assess the performance of imputation algorithms. Based on the type of information used in the index, we categorized these three indices into three different types: (i) statistic index, (ii) clustering index and (iii) differentially expressed genes index.

\section{(i) Statistic index}

For the statistic index, we used the normalized root mean squared error (NRMSE) to evaluate the performance of the imputation algorithms. Lower the value of the statistic index, better the algorithm performs.

Normalized root mean squared error (NRMSE): NRMSE is a popular index used to evaluate the similarity between the true values and the imputed values [33].

$$
N R M S E=\sqrt{\frac{\text { mean }\left[\left(\mathbf{y}_{\text {guess }}-\mathbf{y}_{\text {answer }}\right)^{2}\right]}{\text { variance }\left[\mathbf{y}_{\text {answer }}\right]}}
$$

where $\mathbf{y}_{\text {guess }}$ and $\mathbf{y}_{\text {answer }}$ are vectors, the elements of $\mathbf{y}_{\text {guess }}$ are the imputed values, the elements of $\mathbf{y}_{\text {answer }}$ are the known answer values, and variance[ $\left.\mathbf{y}_{\text {answer }}\right]$ is the variance of $\mathbf{y}_{\text {answer. }}$

\section{(ii) Clustering index}

An important data analysis in the microarray data is the gene clustering. In this study, $k$-means was used to do gene clustering for the complete datasets and the

Table 2 Missing value imputation methods used in this study

\begin{tabular}{lcc}
\hline Methods & Author & Programming Language \\
\hline K-nearest neighbors (KNN) & Local algorithm & Year \\
Iterative K-nearest-neighbors (IKNN) & Troyanskaya O. & R \\
Sequential K-nearest-neighbors (SKNN) & Bras L.P. & Kim K.Y. \\
Least squares adaptive (LSA) & Bø T.H. & Java \\
Local least squares (LLS) & Kim H. & Matlab \\
Iterative local least squares (ILLS) & Cai Z. & Matlab \\
Sequential local least squares (SLLS) & Zhang X & 2004 \\
\hline & 2005 & 2006 \\
\hline Bayesian principal component analysis (BPCA) & Global algorithm & R \\
Singular value decomposition (SVD) & Oba S. & R \\
\hline
\end{tabular}


imputed datasets. We used cluster pair proportions (CPP) [10] as a clustering index to evaluate the performance of the algorithms. The numbers of clusters for each dataset was 10 . Higher the value of the clustering index, better the algorithm performs.

Cluster Pair Proportions (CPP): A schematic illustration of CPP is showed in Figure 1.

\section{(iii) Differentially expressed genes index}

An important data analysis in the microarray is the identification of differentially expressed genes. In this study, SAM was used to identify differentially expressed genes for the complete dataset and the imputed dataset. We used biomarker list concordance index (BLCI) [47] as the differentially expressed genes index to evaluate the performance of the algorithms.

Biomarker list concordance index (BLCI): A high BLCI value indicates that the list of the significantly differentially expressed genes of the complete data is similar to that of the imputed data. And it also means that the imputed data does not significantly change the result of downstream analysis, so the algorithm has excellent performance. We expect that a good algorithm has a high BLCI value. The BLCI is defined as follows:

$$
B L C I\left(B_{C D}, B_{I D}\right)=\frac{n\left(B_{C D} \cap B_{I D}\right)}{n\left(B_{C D}\right)}+\frac{n\left(B_{C D}^{C} \cap B_{I D}^{C}\right)}{n\left(B_{C D}^{C}\right)}-1,
$$

where $B_{C D}$ is the significantly differentially expressed genes from the complete data, and $B_{I D}$ is the significantly differentially expressed genes from the imputed data. $B_{C D}^{C}$ is the complement set of $B_{C D}$, and $B_{I D}^{C}$ is the complement set of $B_{I D}$.

\section{Results and Discussion}

We used (i) thirteen different datasets coming from two organisms (human and yeast), (ii) 110 independent rounds per experiment, and (iii) three kinds of indices to assess nine different algorithms. We thought that the performances of algorithms should be evaluated using measures which can reflect the impact of imputation on downstream analysis. The cluster pair proportions (CPP) is used to assess the results of clustering analysis and the biomarker list concordance index (BLCI) is used to assess the results of identifying differentially expressed genes. Therefore, we used not only normalized root mean squared error (NRMSE), but also CPP and BLCI to evaluate the performance of each algorithm. Such a comprehensive comparison can provide an explicit direction for practitioners and researchers for advanced studies.

\section{Simulation setting}

In our numerical experiments, thirteen real microarray datasets were used as benchmark datasets and nine algorithms including KNN, SKNN, IKNN, LS, LLS, ILLS, SLLS, BPCA and SVD were used.

First, we removed genes with one or more missing values from the original datasets to generate complete data matrices. Second, multiple entries with different

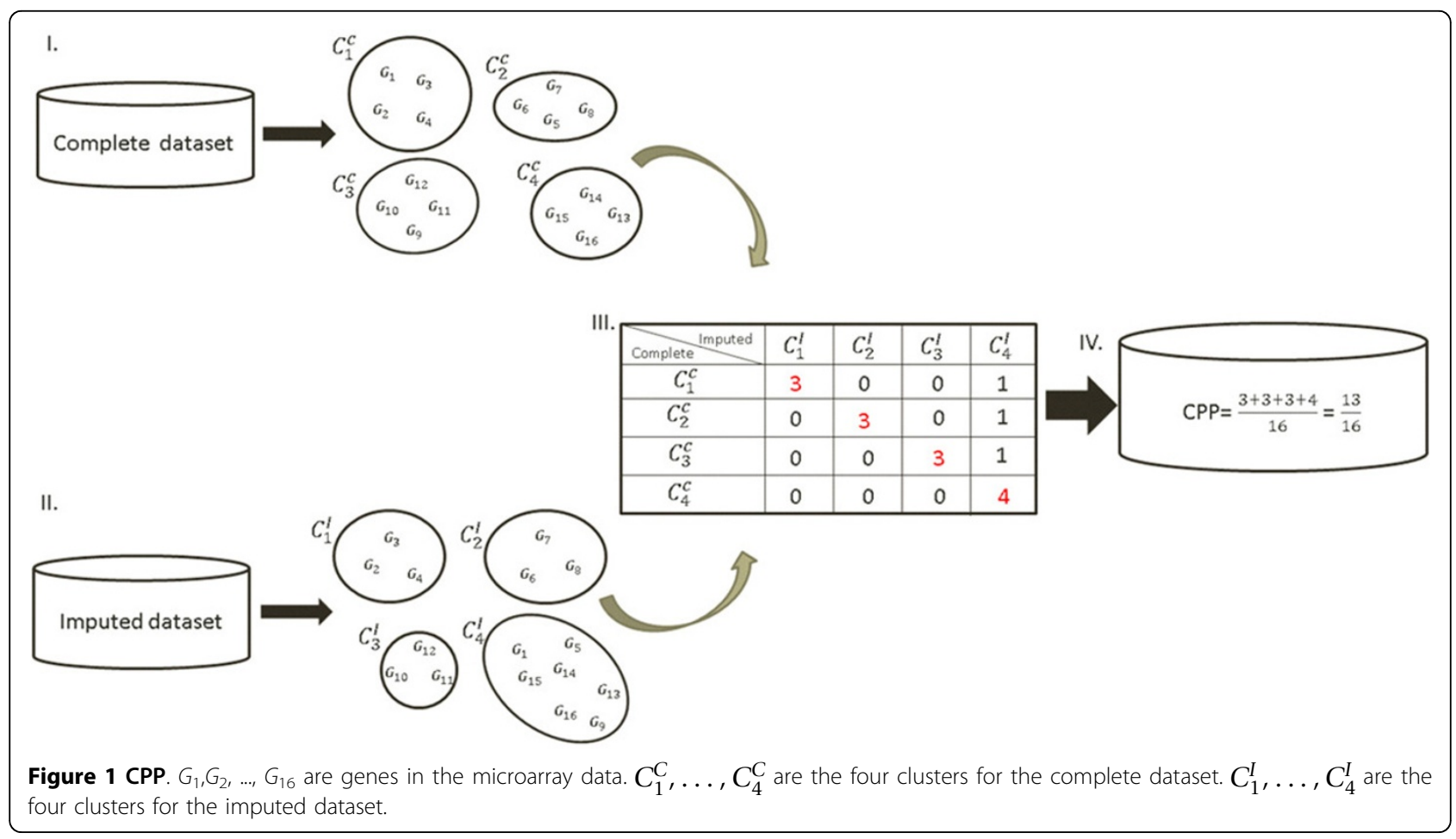


missing percentages $(1 \%, 5 \%, 10 \%, 15 \%$ and $20 \%)$ were randomly introduced into these complete data matrices. And then, the data with missing values was imputed by nine algorithms, respectively. The three steps mentioned above are repeated 110 times for each algorithm. Finally, downstream analysis results from the complete data are compared to the results from the imputed data using three kinds of indices. The workflow of numerical experiments is shown in Figure 2.

\section{The performances of imputation algorithms}

We present a distinct illustration that can point out the optimal method for the microarray datasets used. The $\mathrm{x}$-axis means the algorithms used and the $\mathrm{y}$-axis means the average rank of each algorithm. For example, if we perform an experiment with 5 independent rounds, in which ranks of an algorithm are 1, 2, 2, 1 and 2 respectively. The average rank of the algorithm in this experiment is $(1+2+2+1+2) / 5=1.6$. Thus, in Figure 3a, the average rank of SLLS is 1.4 , which is the result from 110 rounds in an experiment. The error bar for each algorithm is the standard error of the rank.

In this paper, we compared the performances of imputation algorithms using microarrays of various data types to determine the optimal algorithm. Time series, nontime series and mixed type datasets were used as benchmark datasets, and the performance of each algorithm was evaluated using different measures mentioned above. Furthermore, robustness of an imputation algorithm was also disscussed. We compared robustness of an algorithm between various conditions, such as types of datasets and datasets from samples of different organisms.

\section{The ranking of imputation algorithms for different data} types

\section{Performance of imputation algorithms on time series data}

In Figure 4, LLS-like algorithms (based on local least squares methods, such as LLS [23], ILLS [24] and SLLS

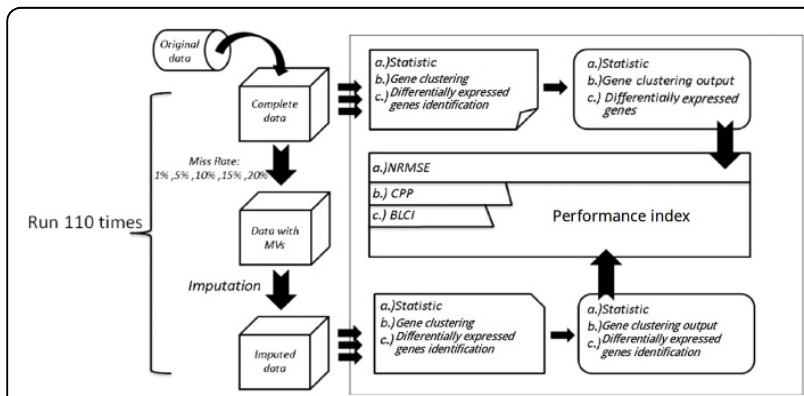

Figure 2 The diagram of the experiment design. (a) is the evaluation using the statistic measure to compare the degree of difference between the complete entries and the imputed entries. (b) and (c) are evaluations using indices with biological meanings to compare the impact of imputation on downstream analysis.
[25]) outperform the others on NRMSE. ILLS is the algorithm with the best performance among the LLSlike algorithms (the average rank $=2.12$ ). The average rank of LS and LLS-like algorithms are around 3.8 using the CPP. SLLS is the optimal method using BLCI (average rank $=2.04$ ).

The performances (average rank) of algorithms are estimated by different indices. The optimal algorithm is ILLS using NRMSE (average rank $=2.12$ ), the optimal algorithms are ILLS and LLS using CPP (average rank = 3.56) and the optimal algorithm is SLLS using BLCI (average rank $=2.04)$. To precisely understand the performances of the algorithms on time series datasets, we averaged each average rank of the algorithms using the different indices as the average rank of the algorithms using the average index on time series datasets. The performance of LLS-like algorithms perform well using the average index. The top two of LLS-like algorithms are SLLS and ILLS. The average rank of SLLS is 2.76 and the average rank of ILLS is 2.79 .

Performance of imputation algorithms on non-time series data

For non-time series datasets (Figure 5), it is prominent that the performance of LS is the best using NRMSE. The average rank of LS is 1.17. Using BLCI, the three algorithms (SKNN, KNN and LS) have the best performance. The average rank of SKNN is 3.23 , the average rank of KNN is 3.37 and the average rank of LS is 3.37. The top performing algorithm is SKNN using CPP. The average rank of SKNN is 3.67. In Figure 5, LS is the optimal algorithm using the average index and then is KNN-based algorithms, such as KNN [17], IKNN [20] and SKNN [21]. We can clearly see that LLS-like algorithms have better performance on time series datasets than on the non-time series datasets.

\section{Performance of imputation algorithms on mixed type data}

In Figure 6, we can obviously see that LS has a low average rank (1.68) using NRMSE. However, the performance of LLS-like algorithms is better than that of LS using BLCI. Using CPP, the average rank of LS is 3.7, the average rank of ILLS is 3.9 , the average rank of KNN is 4.08 and the average rank of SLLS is 4.54. The top three performing algorithms (ILLS, LS and SLLS) are all very competitive with each other. The top performing algorithm is ILLS, followed by LS and SLLS.

Performance of imputation algorithms on all data Performance of each algorithm using the three kinds of indices and the average index on all datasets is given in Figure 7. It can be clearly seen that the performances of LLS-like algorithms and LS are better than the performances of KNN-like algorithms. We noted that no algorithm can perform well on all kinds of datasets. Therefore, the best algorithm cannot be found, but we can find the optimal algorithm for each data type (shown in Table 3). 

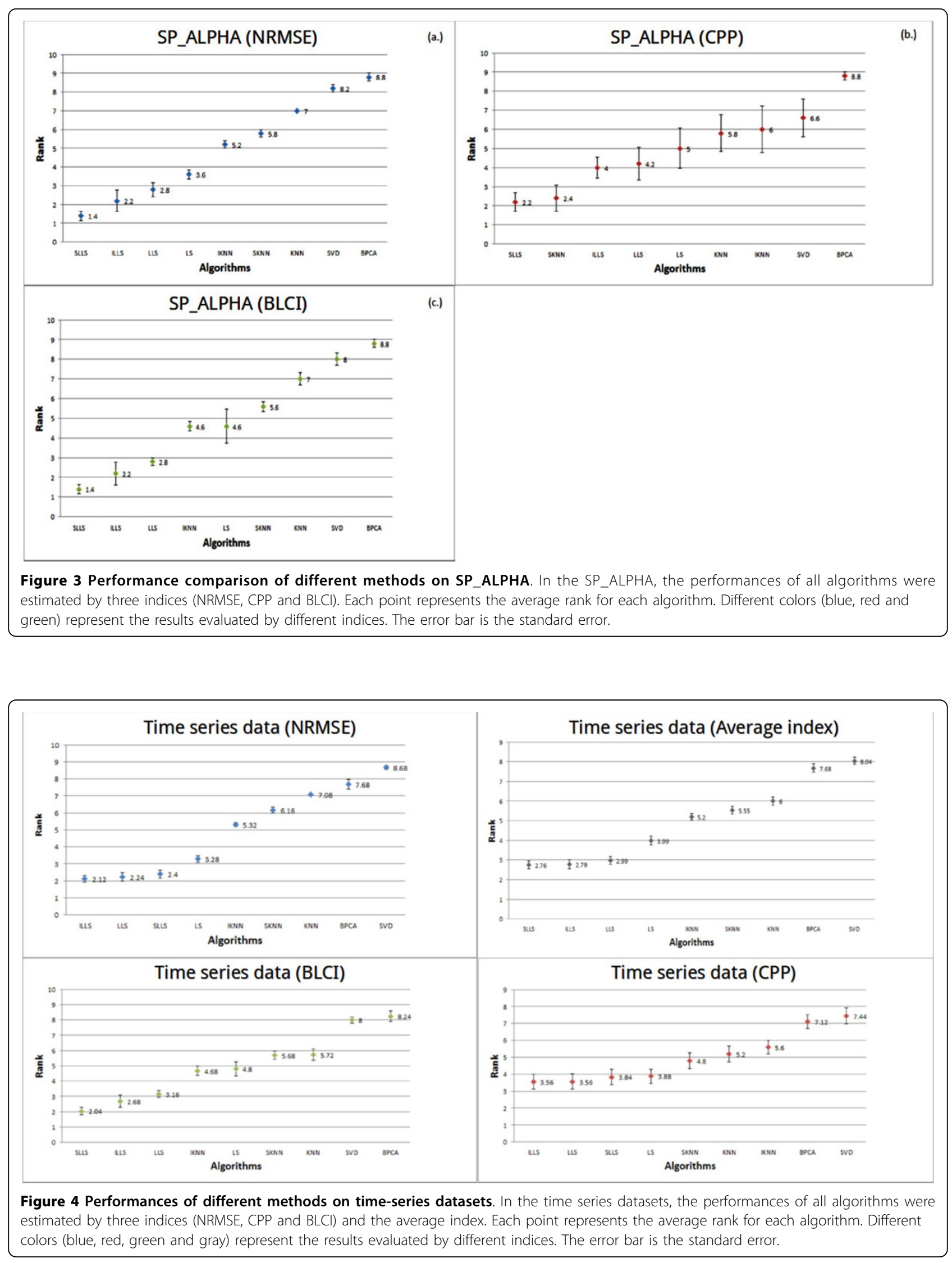

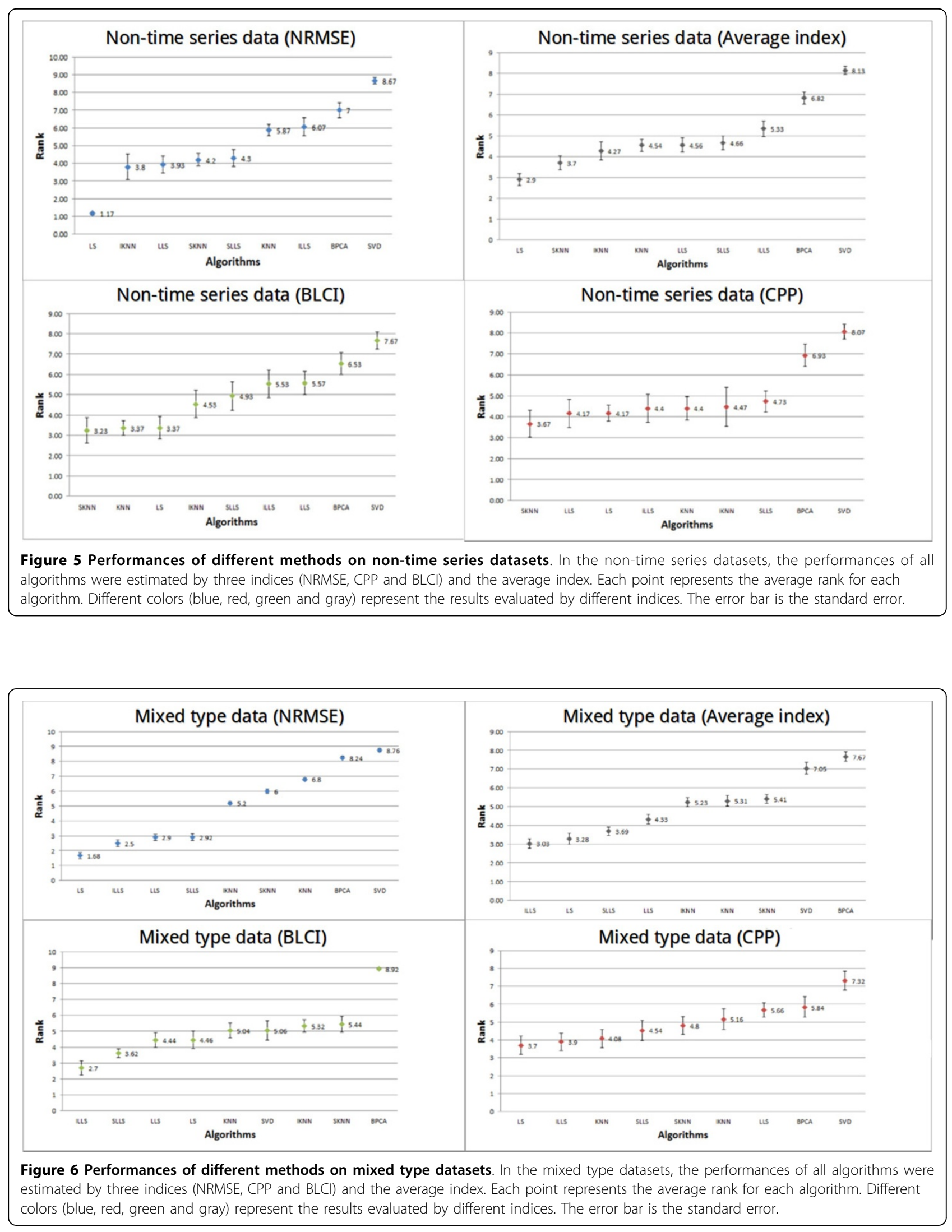


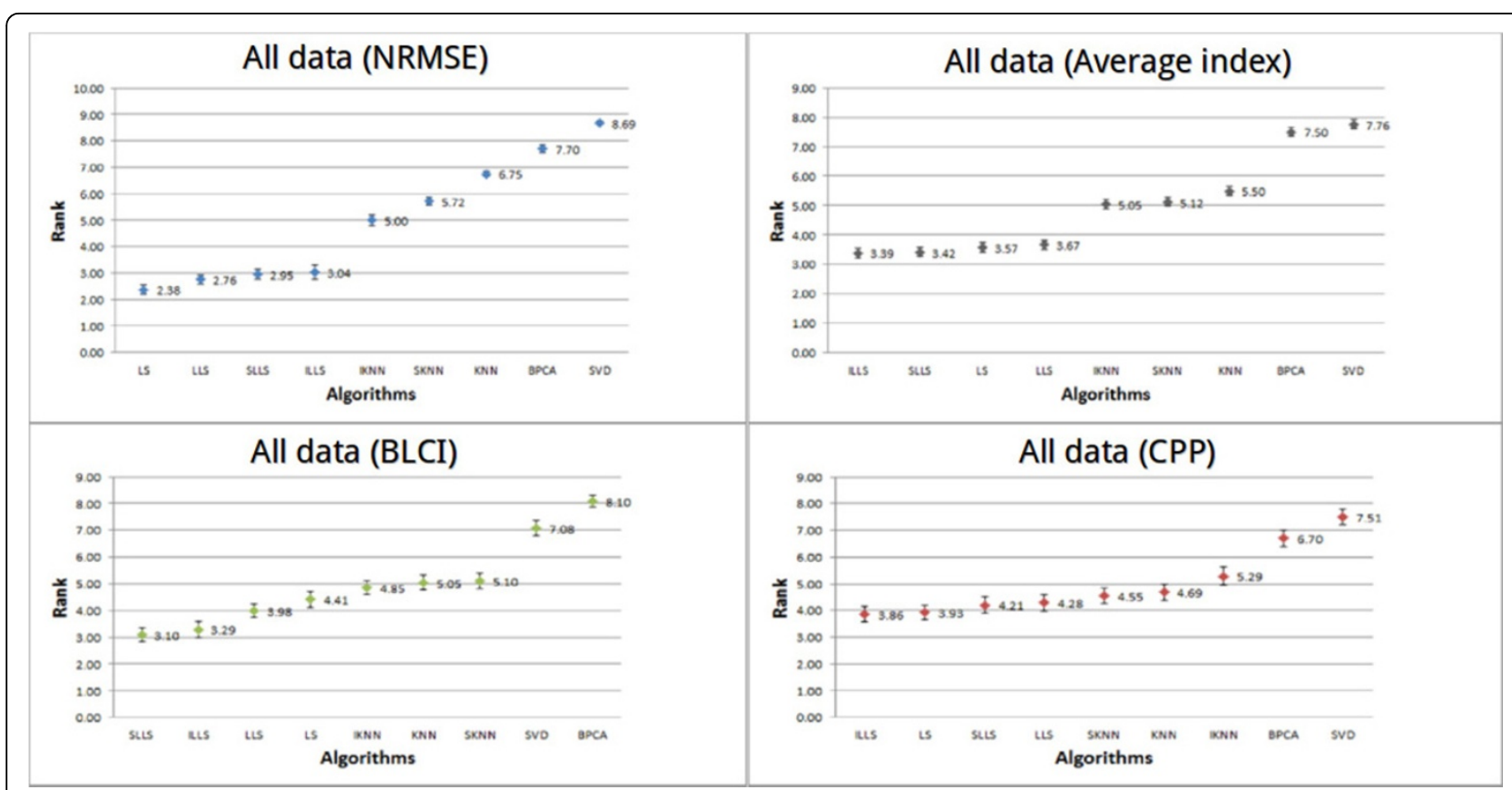

Figure 7 Performances of different methods on all datasets. In all datasets (thirteen datasets), the performances of all algorithms were estimated by three indices (NRMSE, CPP and BLCI) and the average index. Each point represents the average rank for each algorithm. Different colors (blue, red, green and gray) represent the results evaluated by different indices. The error bar is the standard error.

\section{Robustness of each imputation algorithm}

Tuikkala et al. demonstrated that BPCA is the best imputation method on most of datasets [33], while de Brevern et al. indicated that KNN constitutes one efficient method for restoring the missing values with a low error level [10]. According to our experiences, BPCA does not always perform well on all benchmark datasets,

Table 3 The optimal algorithm determined by using various indices for different types of datasets.

\begin{tabular}{clc}
\hline Index & Data & Best algorithm \\
\hline NRMSE & Time series & ILLS \\
& Non-time series & LS \\
& Mixed type & LS \\
& All Data & LS \\
\hline CPP & Time series & ILLS, LLS \\
& Non-time series & SKNN \\
& Mixed type & LS \\
& All data & ILLS \\
\hline$B L C l$ & Time series & SLLS \\
& Non-time series & SKNN \\
& Mixed type & ILLS \\
& All data & SLLS \\
\hline Average index & Time series & SLLS \\
& Non-time series & LS \\
& Mixed type & ILLS \\
& All data & ILLS \\
\hline
\end{tabular}

and the performance of KNN is usually worse than that of other methods for most of time, which means that KNN cannot accurately estimate missing values to improve downstream analysis. Integrating the results of the previous studies with our experiences, it strongly suggests that the optimal imputation algorithms for different types of datasets may be different. Therefore, it is necessary to compare the robustness of each imputation method, which is useful for choosing an optimal algorithm for most of the researchers, especially when they cannot ensure the type of their dataset.

Robustness against different data types

LS outperforms other algorithms using NRMSE (in Figure $8 d$ ) and the average index (in Figure 8a). In Figure $8 \mathrm{a}$ and $8 \mathrm{~d}$, ILLS and SKNN are more sensitive than the other algorithms. When illustration has no explicit trend, we set a threshold $\sigma$ ( $\sigma=\mid$ (non-time series average rank) - (mixed type average rank) $\mid$ ). When $\sigma$ is less than 1.5 , it indicates that the performance of an algorithm is not much different between datasets. In Figure $8 \mathrm{c}$, the performance is not much different between LLS-like algorithms and KNN-like algorithms in mixed type dataset against non-time series dataset. In Figure $8 \mathrm{~b}$, LS, LLS, IKNN, KNN and SLLS are also not much different. On the other hand, ILLS, SKNN, BPCA and SVD are sensitive algorithms. Therefore, in Figure $8 \mathrm{~b}$ and $8 \mathrm{c}$, we suggest that LS can be used when researchers cannot ensure the type of their dataset. 


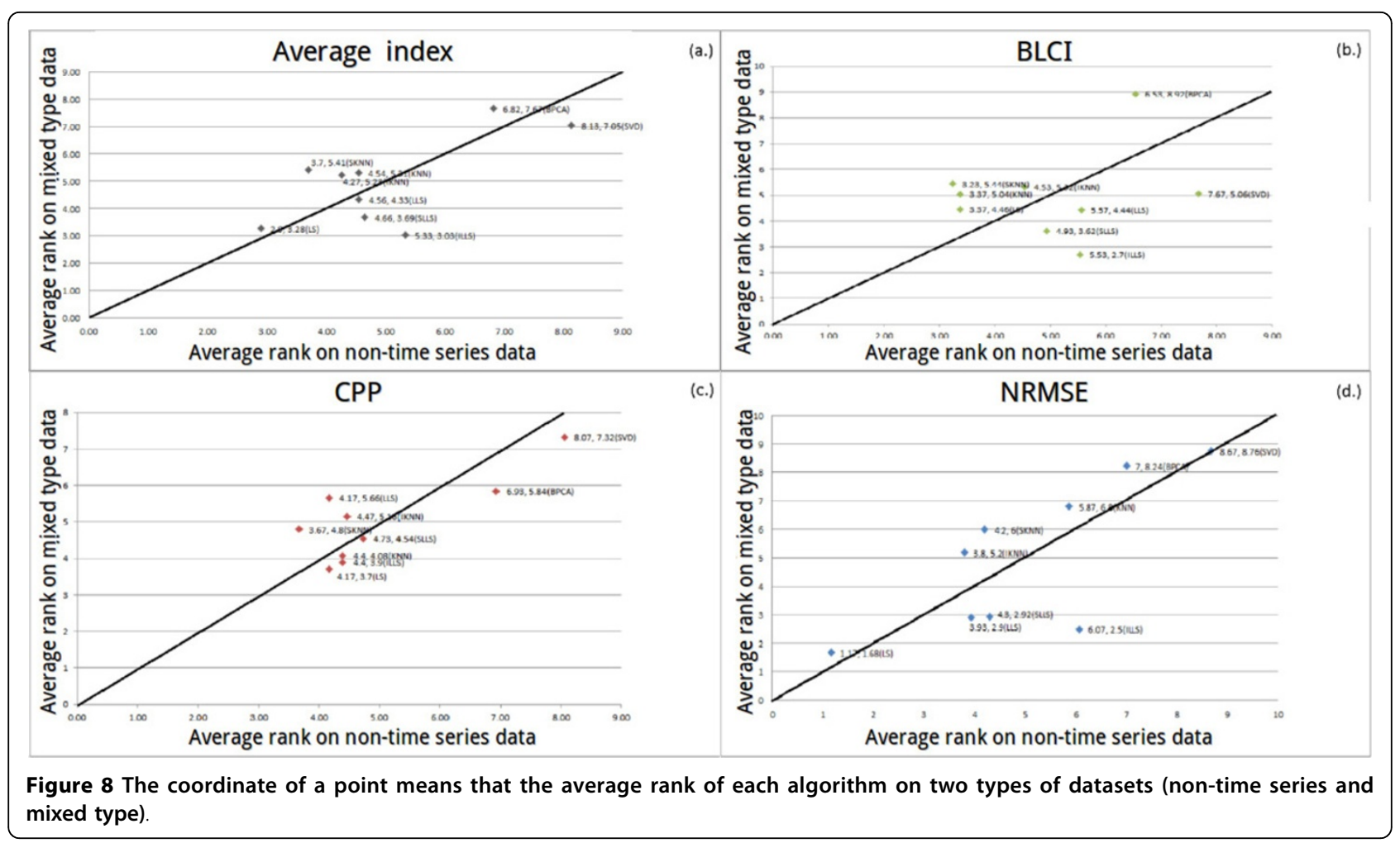

There is an obvious trend in Figure 9a and 9d. Hence, we recommend that LS can be used when researchers cannot ensure whether their dataset belongs to time series dataset or non-time series dataset. In Figure 9c, LS is the optimal algorithm ( $\sigma$ is less than 1.5 and the algorithm is close to left-down) when researchers cannot ensure the type of their datasets. In Figure 9b, LS is still the best one when the type of the dataset is unknown. In Figure 9d, it can be obviously seen that ILLS and LS are more sensitive than the other algorithms. In Figure 9a, LLS-like algorithms prefer time series datasets but not non-time series datasets. SKNN prefer non-time series datasets but not time series datasets. In Figure 9b, ILLS, SLLS and LLS prefer time series datasets but not nontime series datasets. KNN and SKNN prefer non-time series datasets but not time series datasets. In Figure 10c, LLS is more sensitive than the other algorithms. In Figure 10b, SVD prefers mixed type datasets but not time series datasets. In Figure 10a and 10b, ILLS is considered as the optimal algorithm, which can be used when the type of the dataset is either time series or mixed type. In Figure $10 \mathrm{~d}$, the performances of all algorithms are similar between LS and LLS-like algorithms, but LS is still more sensitive than other algorithms. In Figure 10c, ILLS and LS have better performances than the other algorithms.

\section{Robustness against data from different species}

From Figure 11a to $11 \mathrm{~d}$, we can see that $\sigma$ is almost less than 1 for each point $(\sigma=\mid$ Human average rank - Yeast average rank $\mid$ ). This indicates that the performance of each algorithm between different organisms is very similar.

An easy-to-use web tool for missing value imputation

In addition to a comprehensive comparison between imputation algorithms, we developed a web-based imputation tool-MissVIA to help researchers, who do not have good programming skills, to deal with missing values in their datasets. In MissVIA, many existing imputation algorithms were integrated together. MissVIA is built up based on the easy-to-use principle, so every imputation task could be completed with only three steps: (a) upload the dataset with missing values, (b) choose the imputation algorihtms and (c) click the "Submit" button. Once MissVIA receives the request of an imputation task, it will send an e-mail notice with the link of the job to users. Subsequently, MissVIA will initiate a simulation procedure for performance comparison to find out the optimal algorithm (see Figure 12). Finally, the results of performance comparison would be presented with a missing rate-to-NRMSE plot (see Figure 13). According to the plot, MissVIA would determine the optimal algorithm, and then users can use the imputed result for the downstream analysis.

\section{Conclusions}

To find an optimal method to solve the missing value problem efficiently, we conducted a comprehensive 

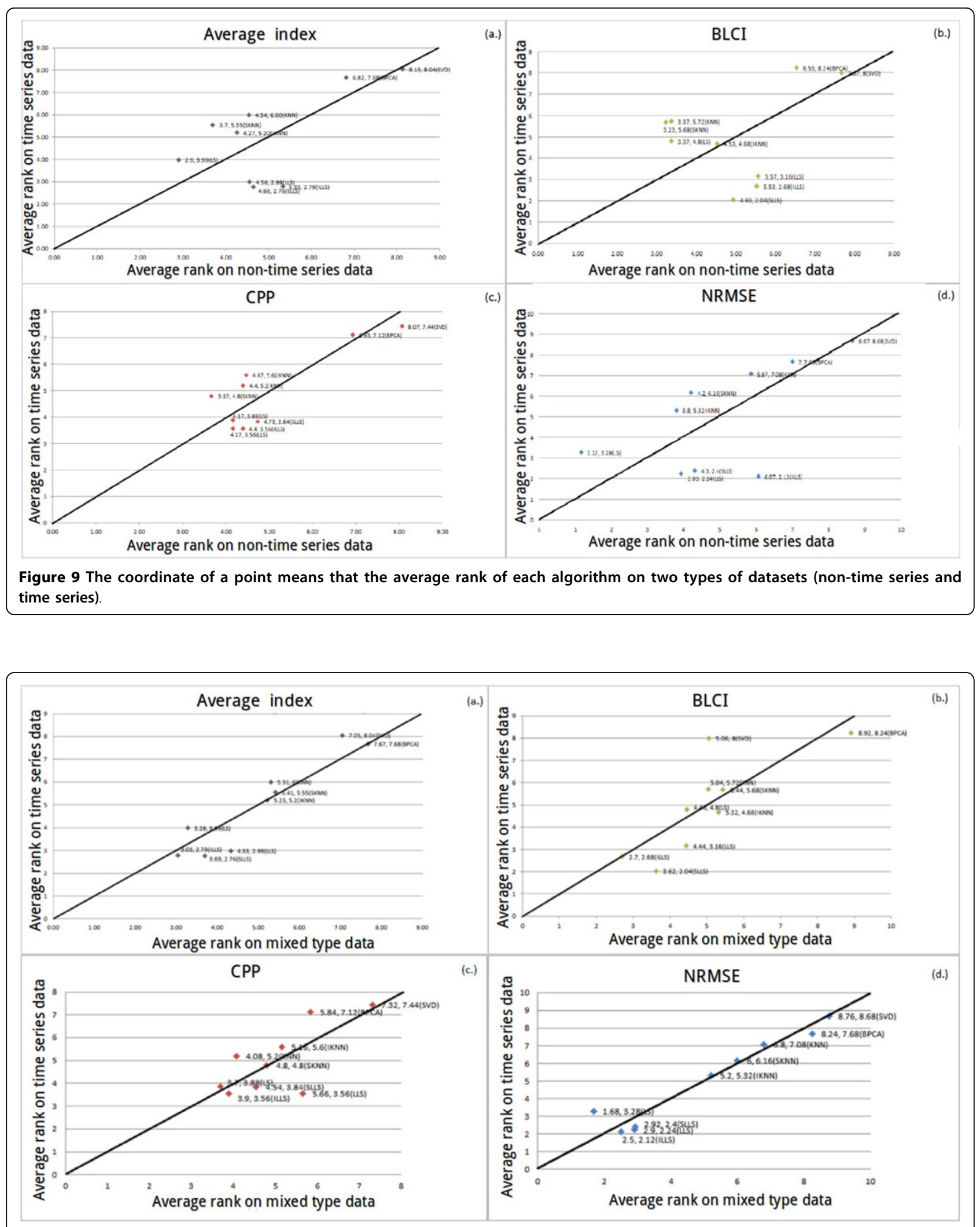

Figure 10 The coordinate of a point means that the average rank of each algorithm on two types of datasets (mixed type and time series dataset). 


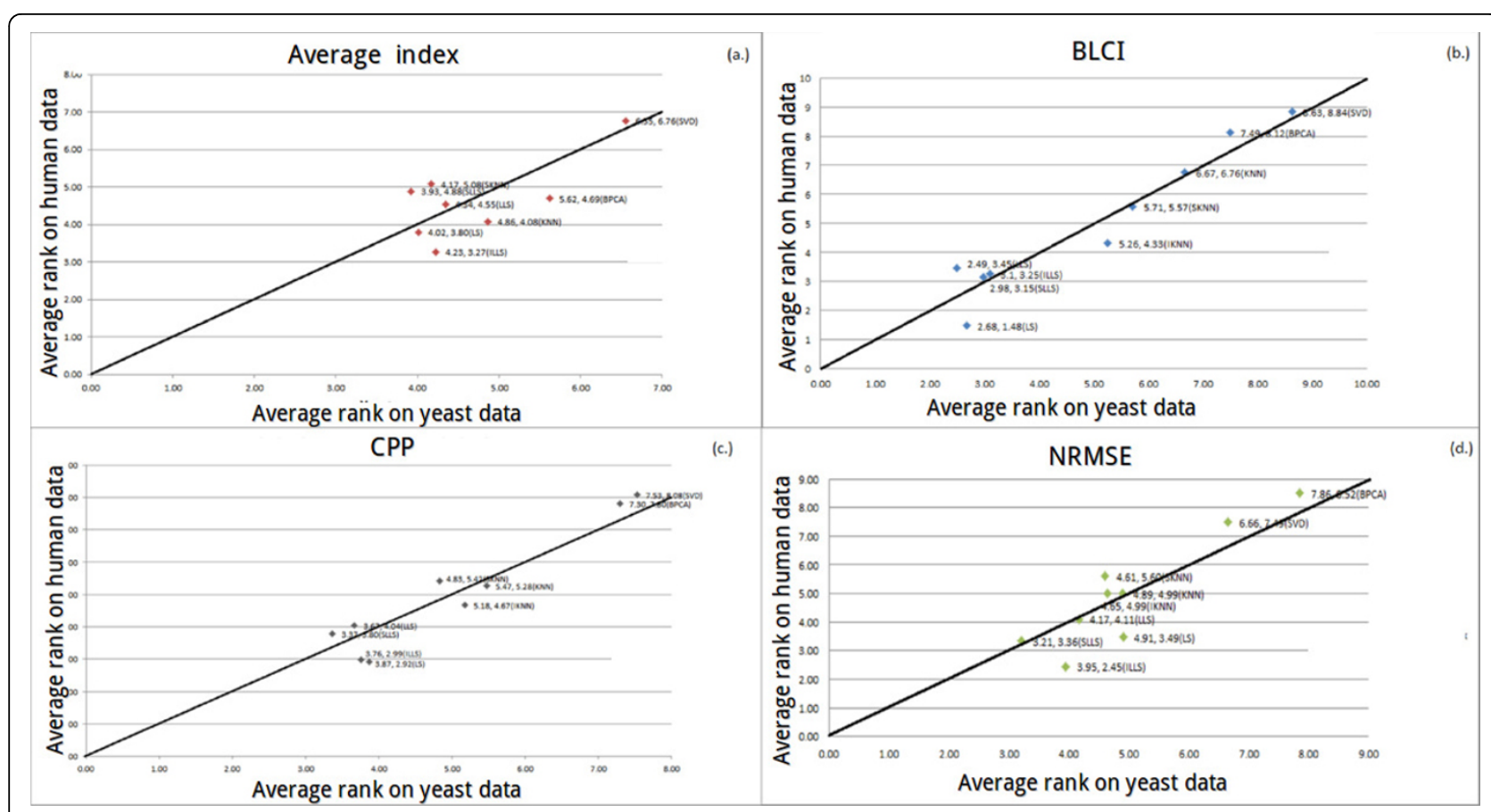

Figure 11 The coordinate of a point means that the average rank of each algorithm on two organisms (yeast and human)

performance comparison of various missing value imputation algorithms in this work. First, we investigated the impact of different types of microarray data on the performance of imputation methods. Three types of microarray data (time series, non-time series and mixed type) were used as benchmark datasets, and the performance

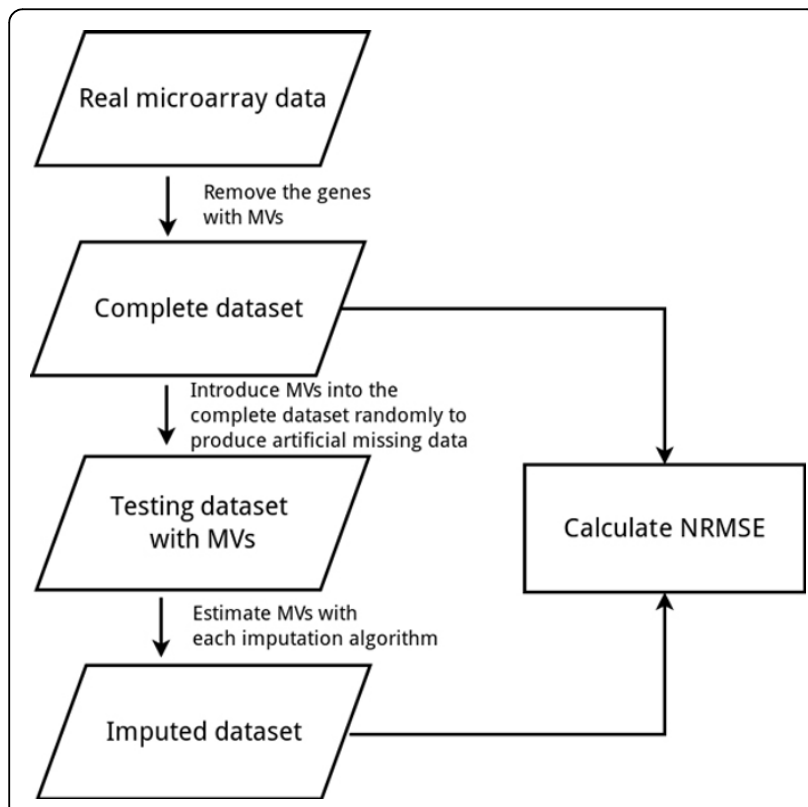

Figure 12 The workflow of performance comparison in MissVIA. of each algorithm was evaluated using three kinds of measures (NRMSE, CPP and BLCI) and the average of these measures (called the average index). These measures are originally used for different purposes. NRMSE is for estimation of deviation between the estimated values and the real values, CPP is for evaluation of clustering results, and BLCI is for assessing the results of finding differentially expressed genes. Our results suggest that, for time series data, ILLS and SLLS have better performances if one wants to do clustering analysis or find differentially expressed genes. For non-time

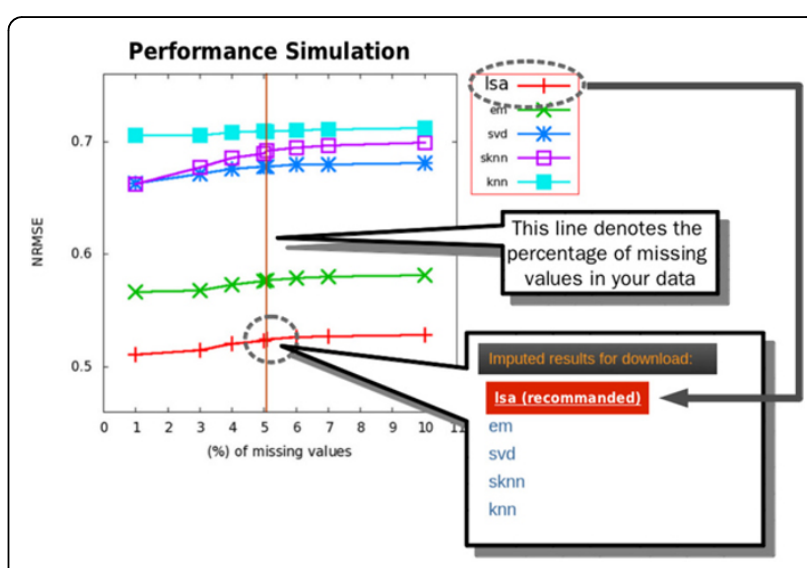

Figure 13 The plot of various missing rates vs. NRMSE generated by MissVIA through the procedure of performance comparison. 
series data, LS is the best algorithm when the performance is evaluated using NRMSE, while SKNN is better than the others if one wants to conduct downstream microarray data analysis. For mixed type data, ILLS is the best choice if one wants to find differentially expressed genes, but LS would be better for the other two purposes.

Then we investigated whether the microarray data from different species would affect the performance of various imputation methods or not. Our results indicate that what kind of species a dataset comes from does not have any obvious effect on the performance of imputation methods. This means that when one is dealing with missing values, what he needs to consider is not the species that the dataset comes from, but the type of the dataset. Besides, we used a distinct illustration to display the relationship between different types of datasets, which is helpful to reveal the robustness of these imputation methods and is useful for researchers to choose an optimal algorithm for their datasets. Besides, to assist experiment practioners in solving missing value problems directly before data analysis, we developed a webbased imputation tool. In this web tool, only 3 steps are needed, and then users could easily obtain a complete dataset imputed by the optimal algorithm.

\section{Competing interests}

The authors declare that they have no competing interests.

\section{Authors' contributions}

WSW conceived the research topic and provided essential guidance. CCC developed the web-based tool, and he did all the simulations with SYC. CCC, SYC, and WSW wrote the manuscript. CCW helps to revise the manuscript. All authors have read and approved the final manuscript.

\section{Acknowledgements}

This study was supported by the National Cheng Kung University and Taiwan National Science Council NSC 99-2628-B-006-015-MY3.

\section{Declarations}

The full funding for the publication fee came from Taiwan National Science Council and College of Electrical Engineering and Computer Science, National Cheng Kung University.

This article has been published as part of BMC Systems Biology Volume 7 Supplement 6, 2013: Selected articles from the 24th International Conference on Genome Informatics (GIW2013). The full contents of the supplement are available online at http://www.biomedcentral.com/bmcsystbiol/supplements/ $7 / 56$.

Published: 13 December 2013

\section{References}

1. Wu W, Li W, Chen B: Computational reconstruction of transcriptional regulatory modules of the yeast cell cycle. BMC Bioinformatics 2006, 7:421.

2. Rowicka M, Kudlicki A, Tu B, Otwinowski Z: High-resolution timing of cell cycle-regulated gene expression. Proc Natl Acad Sci USA 2007, 104:16892-16897.

3. Wu W, Li W, Chen B: Identifying regulatory targets of cell cycle transcription factors using gene expression and ChIP-chip data. BMC Bioinformatics 2007, 8:188.
4. Futschik M, Herzel $\mathrm{H}$ : Are we overestimating the number of cell-cycling genes? The impact of background models on time-series analysis. Bioinformatics 2008, 24:1063-1069.

5. Wu W, Li W: Systematic identification of yeast cell cycle transcription factors using multiple data sources. BMC Bioinformatics 2008, 9:522.

6. Siegal-Gaskins D, Ash J, Crosson S: Model-based deconvolution of cell cycle time-series data reveals gene expression details at high resolution. PLoS Comput Biol 2009, 5:e1000460.

7. Wang $\mathrm{H}$, Wang $\mathrm{Y}, \mathrm{Wu}$ W: Yeast cell cycle transcription factors identification by variable selection criteria. Gene 2011, 485:172-176.

8. Gasch A, Spellman P, Kao C, Carmel-Harel O, Eisen M, Storz G, Botstein D, Brown P: Genomic expression programs in the response of yeast cells to environmental changes. Mol Biol Cell 2000, 11:4241-4257.

9. Wu W, Li W: Identifying gene regulatory modules of heat shock response in yeast. BMC Genomics 2008, 9:439.

10. de Brevern AG, Hazout S, Malpertuy A: Influence of microarrays experiments missing values on the stability of gene groups by hierarchical clustering. BMC Bioinformatics 2004, 5:114

11. Jörnsten R, Wang HY, Welsh WJ, Ouyang M: DNA microarray data imputation and significance analysis of differential expression. Bioinformatics 2005, 21(22):4155-4161.

12. Scheel I, Aldrin M, Glad IK, Sørum R, Lyng H, Frigessi A: The influence of missing value imputation on detection of differentially expressed genes from microarray data. Bioinformatics 2005, 21(23):4272-4279.

13. Sehgal MSB, Gondal I, Dooley LS, Coppel R: How to improve postgenomic knowledge discovery using imputation. EURASIP Journal on Bioinformatics and Systems Biology 2009, 2009:717136.

14. Zhang Y, Xuan J, Reyes BGdl, Clarke R, Ressom HW: Reverse engineering module networks by PSO-RNN hybrid modeling. BMC Genomics 2009, 10(Suppl 1):S15

15. Wang D, Lv Y, Guo Z, Li X, Li Y, Zhu J, Yang D, Xu J, Wang C, Rao S, Yang B: Effects of replacing the unreliable cDNA microarray measurements on the disease classification based on gene expression profiles and functional modules. Bioinformatics 2006, 22(23):2883-2889.

16. Ouyang M, Welsh WJ, Georgopoulos P: Gaussian mixture clustering and imputation of microarray data. Bioinformatics 2004, 20(6):917-923.

17. Troyanskaya O, Cantor M, Sherlock G, Brown P, Hastie T, Tibshirani R, Botstein D, Altman RB: Missing value estimation methods for DNA microarrays. Bioinformatics (Oxford, England) 2001, 17(6):520-525.

18. Liew AWC, Law NF, Yan H: Missing value imputation for gene expression data: computational techniques to recover missing data from available information. Briefings in bioinformatics 2011, 12(5):498-513.

19. Moorthy K, Mohamad MS, Deris S: A review on missing value imputation algorithms for microarray gene expression data. Advance in Bioinformatics 2013.

20. Brãs LP, Menezes JC: Improving cluster-based missing value estimation of DNA microarray data. Biomolecular engineering 2007, 24(2):273-282.

21. Kim KY, Kim BJ, Yi GS: Reuse of imputed data in microarray analysis increases imputation efficiency. BMC Bioinformatics 2004, 5:160.

22. $B \varnothing T H$, Dysvik $B$, Jonassen I: LSimpute: accurate estimation of missing values in microarray data with least squares methods. Nucleic Acids Research 2004, 32(3):e34.

23. Kim H, Golub GH, Park H: Missing value estimation for DNA microarray gene expression data: local least squares imputation. Bioinformatics 2005, 21(2):187-198.

24. Cai Z, Heydari M, Lin G: Iterated local least squares microarray missing value imputation. Journal of bioinformatics and computational biology 2006, 4(5):935-957.

25. Zhang $X$, Song $X$, Wang $H$, Zhang $H$ : Sequential local least squares imputation estimating missing value of microarray data. Computers in biology and medicine 2008, 38(10):1112-1120.

26. Oba S, Sato Ma, Takemasa I, Monden M, Matsubara Ki, Ishii S: A Bayesian missing value estimation method for gene expression profile data. Bioinformatics 2003, 19(16):2088-2096.

27. Jelizarow M, Guillemot V, Tenenhaus A, Strimmer K, Boulesteix AL: Overoptimism in bioinformatics: an illustration. Bioinformatics 2010, 26(16):1990-1998.

28. Gan X, Liew AWC, Yan H: Microarray missing data imputation based on a set theoretic framework and biological knowledge. Nucleic Acids Research 2006, 34(5):1608-1619. 
29. Xiang Q, Dai X, Deng Y, He C, Wang J, Feng J, Dai Z: Missing value imputation for microarray gene expression data using histone acetylation information. BMC Bioinformatics 2008, 9:252.

30. Johansson P, Häkkinen J: Improving missing value imputation of microarray data by using spot quality weights. BMC Bioinformatics 2006, 7:306

31. Hu J, Li H, Waterman MS, Zhou XJ: Integrative missing value estimation for microarray data. BMC Bioinformatics 2006, 7:449.

32. Brock GN, Shaffer JR, Blakesley RE, Lotz MJ, Tseng GC: Which missing value imputation method to use in expression profiles: a comparative study and two selection schemes. BMC Bioinformatics 2008, 9:12.

33. Tuikkala J, Elo LL, Nevalainen OS, Aittokallio T: Missing value imputation improves clustering and interpretation of gene expression microarray data. BMC Bioinformatics 2008, 9:202.

34. Celton M, Malpertuy A, Lelandais G, Brevern AGd: Comparative analysis of missing value imputation methods to improve clustering and interpretation of microarray experiments. BMC Genomics 2010, 11:15.

35. Rao SSS, Shepherd LA, Bruno AE, Liu S, Miecznikowski JC: Comparing imputation procedures for Affymetrix gene expression datasets using MAQC datasets. Current Bioinformatics 2013.

36. Spellman PT, Sherlock G, Zhang MQ, lyer VR, Anders K, Eisen MB, Brown PO, Botstein D, Futcher B: Comprehensive identification of cell cycleregulated genes of the yeast Saccharomyces cerevisiae by microarray hybridization. Molecular Biology of the Cell 1998, 9(12):3273-3297.

37. Shapira M, Segal E, Botstein D: Disruption of yeast forkhead-associated cell cycle transcription by oxidative stress. Molecular Biology of the Cell 2004, 15(12):5659-5669.

38. Whitfield ML, Sherlock G, Saldanha AJ, Murray JI, Ball CA, Alexander KE, Matese JC, Perou CM, Hurt MM, Brown PO, Botstein D: Identification of genes periodically expressed in the human cell cycle and their expression in tumors. Molecular biology of the cell 2002, 13(6):1977-2000.

39. Ogawa N, DeRisi J, Brown PO: New components of a system for phosphate accumulation and polyphosphate metabolism in Saccharomyces cerevisiae revealed by genomic expression analysis. Molecular biology of the cell 2000, 11(12):4309-4321.

40. Bohen SP, Troyanskaya OG, Alter O, Warnke R, Botstein D, Brown PO, Levy R: Variation in gene expression patterns in follicular lymphoma and the response to rituximab. Proceedings of the National Academy of Sciences of the United States of America 2003, 100(4):1926-1930.

41. Alizadeh AA, Eisen MB, Davis RE, Ma C, Lossos IS, Rosenwald A, Boldrick JC, Sabet H, Tran T, Yu X, Powell Jl, Yang L, Marti GE, Moore T, Hudson JJ, Lu L, Lewis DB, Tibshirani R, Sherlock G, Chan WC, Greiner TC, Weisenburger DD, Armitage JO, Warnke R, Levy R, Wilson W, Grever MR, Byrd JC, Botstein D, Brown PO, Staudt LM: Distinct types of diffuse large B-cell lymphoma identified by gene expression profiling. Nature 2000, 403(6769):503-511.

42. Baldwin DN, Vanchinathan V, Brown PO, Theriot JA: A gene-expression program reflecting the innate immune response of cultured intestinal epithelial cells to infection by Listeria monocytogenes. Genome Biology 2002, 4:R2.

43. Yoshimoto H, Saltsman K, Gasch AP, Li HX, Ogawa N, Botstein D, Brown PO, Cyert MS: Genome-wide analysis of gene expression regulated by the calcineurin/Crz1p signaling pathway in Saccharomyces cerevisiae. The Journal of biological chemistry 2002, 277(34):31079-31088.

44. Brauer MJ, Saldanha AJ, Dolinski K, Botstein D: Homeostatic adjustment and metabolic remodeling in glucose-limited yeast cultures. Molecular Biology of the Cell 2005, 16(5):2503-2517.

45. Ronen M, Botstein D: Transcriptional response of steady-state yeast cultures to transient perturbations in carbon source. Proceedings of the National Academy of Sciences of the United States of America 2006, 103(2):389-394.

46. Sehgal MSB, Gondal I, Dooley LS: Collateral missing value imputation: a new robust missing value estimation algorithm for microarray data. Bioinformatics (Oxford, England) 2005, 21(10):2417-2423.

47. Oh S, Kang DD, Brock GN, Tseng GC: Biological impact of missing-value imputation on downstream analyses of gene expression profiles. Bioinformatics 2011, 27:78-86.

doi:10.1186/1752-0509-7-S6-S12

Cite this article as: Chiu et al.: Missing value imputation for microarray data: a comprehensive comparison study and a web tool. BMC Systems Biology 2013 7(Suppl 6):S12

\section{Submit your next manuscript to BioMed Central and take full advantage of:}

- Convenient online submission

- Thorough peer review

- No space constraints or color figure charges

- Immediate publication on acceptance

- Inclusion in PubMed, CAS, Scopus and Google Scholar

- Research which is freely available for redistribution 